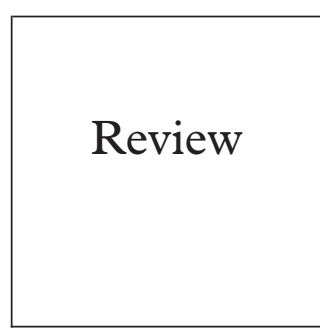

HIV and STD

Division,

Communicable

Disease Surveillance

Centre

I Simms

Department of

Sexually Transmitted

Diseases, University

College London

Medical School

J M Stephenson

Correspondence to:

Mr Ian Simms, PHLS

Communicable Disease

Surveillance Centre, 61

Colindale Avenue, London

NW9 $5 \mathrm{EQ}$

isimms@phls.nhs.uk

Accepted for publication 24 February 2000

\title{
Pelvic inflammatory disease epidemiology: what do we know and what do we need to know?
}

\author{
I Simms, J M Stephenson
}

"Pelvic inflammatory disease is a sexually transmitted disease with potentially serious sequelae usually managed badly by doctors with little interest in the condition."

\section{Introduction}

It is a decade since this bleak view of pelvic inflammatory disease (PID) management in the United Kingdom appeared in the $B M \mathcal{}$. ${ }^{1}$ Since then a theme to emerge in sexually transmitted disease (STD) research has been increased awareness of genital chlamydial infection, which causes a substantial proportion of PID cases. In the United Kingdom, this culminated in the Chief Medical Officer's expert advisory group on genital chlamydial infection which recognised PID as an important source of preventable reproductive morbidity in women. ${ }^{2}$ However, little is known of PID epidemiology in England and Wales. The burden of disease and risk factors associated with PID are poorly understood but need to be investigated to inform public health action and clinical practice. ${ }^{3}$ This paper aims to critically review current knowledge of PID epidemiology with special reference to the United Kingdom and explore the epidemiological research needed to provide an evidence base for PID public health intervention.

\section{Methods}

A literature search was carried out on Medline using the key words "pelvic inflammatory disease" and was repeated using authors known to have published studies concerned with PID and Chlamydia trachomatis. The literature was also trawled for data presentations.

\section{Aetiology of PID}

PID is the clinical syndrome associated with upper genital tract inflammation caused by the spread of micro-organisms from the lower to the upper genital tract. PID can be caused by genital mycoplasmas, endogenous vaginal flora (anaerobic and aerobic bacteria), aerobic streptococci, Mycobacterium tuberculosis, and sexually transmitted infections (STI) such as $C$ trachomatis or Neisseria gonorrhoeae ${ }^{4}$ An association between PID and bacterial vaginosis has also been demonstrated in the absence of $C$ trachomatis and $N$ gonorrhoeae. ${ }^{56}$ A number of aetiological studies have been undertaken over the past 20 years in various clinical settings (table 1 ). In these studies, $C$ trachomatis was detected in $14 \%-65 \%$ of PID cases but, since these are small scale studies, this does not reflect substantial aetiological variation over time and between countries. Some studies report a higher prevalence of $N$ gonorrhoeae than $C$ trachomatis, but again it should be remembered that the studies are based on small sample sizes. ${ }^{78}$ Nevertheless, the studies do indicate that a substantial proportion of PID cases are caused by $C$ trachomatis. The largest UK study, based on only 147 women at one location, indicated that $39 \%$ (95\% CL $29 \%$ to $49 \%$ ) of PID cases were caused by $C$ trachomatis and $14 \%$ were caused by gonorrhoea. $^{9}$ The proportion of PID cases caused by $C$ trachomatis is a vital consideration in any chlamydial intervention programme as the number of PID cases that could be prevented should be estimated before and during intervention. PID aetiology should thus be assessed at the beginning and during any chlamydial intervention programme.

\section{Pathogenesis and spectrum of disease}

PID sequelae include ectopic pregnancy, tubal factor infertility (TFI), and chronic pelvic pain. PID has also been associated with increased risk of ovarian cancer (fig 1). ${ }^{10-12}$ Pathogenesis is a complex interaction of genetic, immunological, and bacterial virulence factors ${ }^{13}$ and

\begin{tabular}{|c|c|c|c|c|c|}
\hline Population & Country & $\begin{array}{l}\text { Prevalence \% } \\
(95 \% C L)\end{array}$ & Sample size & Site of specimen collection & Author (year) reference \\
\hline STD clinics & UK & $40(25-55)$ & $17 / 43$ & Lower genital tract & Kinghorn $(1986)^{77}$ \\
\hline \multirow[t]{9}{*}{ Gynaecology inpatients } & UK & $39(29-49)$ & $40 / 104$ & Lower genital tract and upper genital tract & Bevan $(1995)^{9}$ \\
\hline & UK & $43(23-66)$ & $10 / 23$ & Lower genital tract and upper genital tract & Stacey $(1992)^{78}$ \\
\hline & Finland & $30(24-37)$ & $69 / 228$ & Lower genital tract & Paavonen $(1980)^{79}$ \\
\hline & Finland & $42(26-59)$ & $15 / 36$ & Lower genital tract and upper genital tract & Heinonen $(1989)^{80}$ \\
\hline & Finland & $52(29-63)$ & $16 / 35$ & Lower genital tract and upper genital tract & Paavonen $(1987)^{81}$ \\
\hline & Sweden & $33(26-41)$ & $52 / 156$ & Lower genital tract & Ripa $(1980)^{82}$ \\
\hline & Sweden & $12(7-17)$ & $22 / 187$ & Upper genital tract & Brihmer (1987) ${ }^{83}$ \\
\hline & USA & $15(9-25)$ & $13 / 84$ & Upper genital tract & Soper $(1994)^{84}$ \\
\hline & USA & $22(19-25)$ & $129 / 589$ & Lower genital tract and upper genital tract & Jossens $(1994)^{76}$ \\
\hline \multirow[t]{3}{*}{ Gynaecology outpatients } & Canada & $16(7-29)$ & $8 / 50$ & Lower genital tract and upper genital tract & Brunham $(1988)^{7}$ \\
\hline & Finland & $14(5-26)$ & $7 / 51$ & Upper genital tract & Cacciatore $(1992)^{85}$ \\
\hline & USA & $65(43-84)$ & $15 / 23$ & Lower genital tract and upper genital tract & Wølner-Hanssen $(1988)^{8}$ \\
\hline \multirow{3}{*}{ Accident and emergency } & USA & $61(39-80)$ & $14 / 23$ & Upper genital tract & Wasserheit $(1986)^{86}$ \\
\hline & USA & $38(25-52)$ & $21 / 55$ & Lower genital tract and upper genital tract & Kiviat $(1986)^{87}$ \\
\hline & USA & $30(13-53)$ & $7 / 23$ & Lower genital tract and upper genital tract & Livengood $(1992)^{88}$ \\
\hline Primary care & Canada & $25(13-40)$ & $11 / 44$ & Lower genital tract and upper genital tract & Sellors $(1991)^{69}$ \\
\hline
\end{tabular}




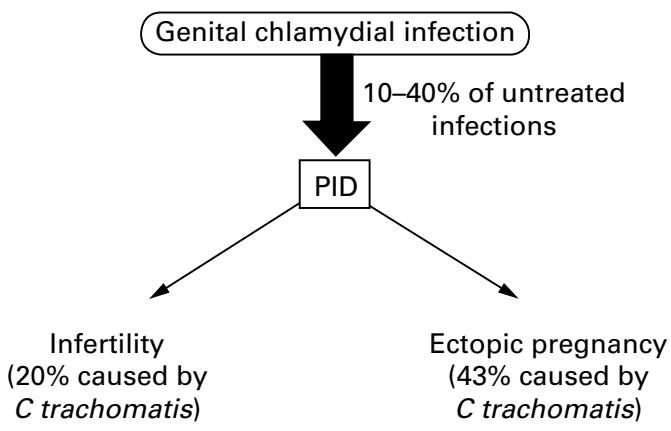

Figure 1 Relation between genital chlamydial infection, pelvic inflammatory disease, and sequelae of pelvic inflammatory disease.

current understanding of the immunopathological pathways from infection to PID and tubal scarring is incomplete. Chronic sequelae of genital chlamydial infection such as ectopic pregnancy and tubal fertility are thought to be caused by a delayed hypersensitivity reaction to chlamydial $60 \mathrm{kDa}$ chlamydial heat shock protein (HSP-60). ${ }^{14-16}$ The clinical presentation and course of PID in women with symptomatic HIV disease and/or severe immune suppression may be more aggressive than in HIV negative women. ${ }^{17}$

Between $10 \%$ and $40 \%$ of $C$ trachomatis cases develop PID. ${ }^{18}$ The risk of developing sequelae is dependent on the number of PID episodes: the risk of ectopic pregnancy and infertility increases after one PID episode (odds ratio 6) and again after two episodes (OR 17). ${ }^{19}$ Animal models indicate that PID can develop within 5 days of $C$ trachomatis infection. ${ }^{20}$ Failure to seek treatment within 3 days of the onset of lower abdominal pain can result in a threefold increase in the risk of PID and infertility. ${ }^{21}$ Early diagnosis is thus essential.

Few studies have investigated the incidence of PID sequelae in women with a history of PID as large patient groups are difficult to follow up over long periods of time. In particular, the prevalence of infertility is difficult to estimate as it is only reported by those who wish to conceive. The definitive study of morbidity associated with PID included 2501 women in Lund (Sweden) between 1960 and 1984. ${ }^{19}$ Data from this study indicate that those women who had a history of PID were six times more likely to have an ectopic pregnancy and 14 times more likely to have tubal factor infertility than women who had no evidence or history of PID. In the United Kingdom, an 11 year record linkage cohort study showed that women with a history of PID were $6,8,10$, and 10 times more likely to have diagnoses of endometritis, hysterectomy, abdominal pain, and ectopic pregnancy, respectively, than controls. ${ }^{11}$

\section{Problems associated with PID surveillance}

The problems associated with PID surveillance stem from the fact that a cheap, simple, and accurate diagnostic test does not exist. No single infection causes PID and no signs and symptoms are pathognomonic of the disease.
These problems of case definition and diagnostic accuracy are compounded by the inaccessibility of the female upper genital tract to routine, large scale diagnostic methods. Consequently it is difficult to formulate a diagnostic "gold standard." PID surveillance data are also influenced by variations in case definitions (particularly between clinical settings), changes in disease chronicity associated with clinically mild chlamydial infection, variations in health seeking behaviour, and the increased management of PID in outpatient settings. ${ }^{22}{ }^{23}$ Variations in the use of intrauterine devices (IUD) may also influence PID prevalence. Swedish data indicate that PID hospital admissions varied by less than $16 \%$ from year to year in the early 1970 s and 1980 s, but increased by $75 \%$ in the mid-1970s, fluctuations that reflected variations in IUD use. ${ }^{24} 25$

Trends in PID cannot be inferred from genital chlamydial infection as the surveillance data are heavily influenced by case ascertainment bias and are thus unrepresentative of the true reservoir of genital chlamydial infection in the general population, a problem seen in the US surveillance data. ${ }^{26}$ The prevalence of $C$ trachomatis cannot be inferred from that of gonorrhoea as these infections have distinctly different epidemiologies. Gonorrhoea prevalence has declined in several European countries over the past 15 years ${ }^{25}{ }^{27}$ whereas a decline in chlamydial prevalence has only been demonstrated in Sweden. ${ }^{25}$

The biases inherent in the surveillance of PID related infections and sequelae make it difficult to assess trends in PID prevalence with certainty. Comparisons between countries are difficult, if not impossible, to make.

\section{Incidence, prevalence, and recent trends} in industrialised countries

Since a substantial proportion of PID cases are caused by STIs, epidemics of $N$ gonorrhoeae and $C$ trachomatis are followed by a secondary PID epidemic and tertiary epidemics of ectopic pregnancy and tubal infertility. An example of these relations is seen in Swedish surveillance data. The epidemic of $N$ gonorrhoeae experienced by industrialised countries in the 1960s peaked in Sweden in 1970, and then decreased. $^{28}$ An associated PID epidemic peaked at 11/1000 women aged 15-39 between 1970 and 1974 and then declined as a tertiary ectopic pregnancy epidemic emerged. ${ }^{10} 29$ The decline in both STIs and first and repeat episodes of PID, together with a change in sexual behaviour brought about by intervention, suggest that the observed decrease in PID prevalence was real. ${ }^{30}$ In $1970,15 \%$ of reproductive age women in Lund reported ever having been treated for PID, similar to the $10 \%$ reported in the United States. ${ }^{30} 31$ The incidence of 14/1000 women aged 14-34 seen in the United States in the same period was also similar to that seen in Sweden. ${ }^{10}{ }^{30}$ Since 1970, the burden of STDs has varied considerably between countries in response to different STI transmission patterns and variations in intervention strategies. 


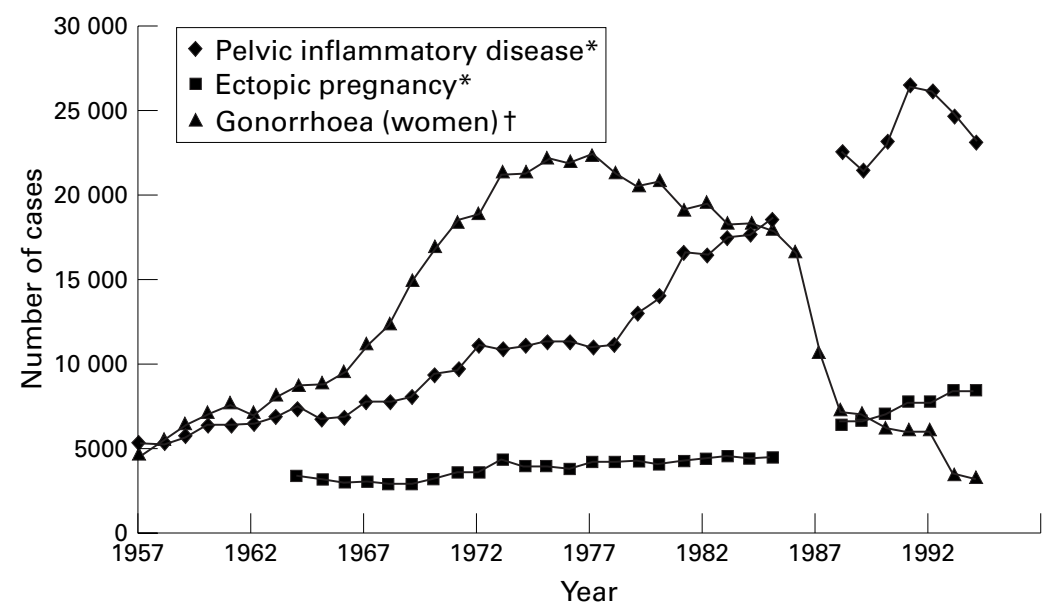

Figure 2 Gonorrhoea, pelvic inflammatory disease, and ectopic pregnancy: England and Wales 1957-94. Hospital inpatient collection method changed after 1985. No data are available for 1986 and 1987. Ectopic pregnancy data are not available before 1964

Although a substantial burden of PID is thought to exist in many countries, surveillance data are only available for a few European countries, mostly in Scandinavia. In England and Wales, variations in gonorrhoea cases seen in STD clinics and hospital inpatient attendances for PID and ectopic pregnancy have followed a pattern similar to that seen in Sweden over the past five decades (fig 2). Interpretation is, however, difficult. There are a number of gaps in reporting caused by either changes in collection methods or absence of data. However, the main concern is that the steep increase in gonorrhoea seen after the second world war is not reflected in a rise in attendances for PID as would be expected. This questions the representativeness and accuracy of attendance data for PID and ectopic pregnancy during the 1950s. Age specific data from 1966 onwards indicates that highest PID prevalence and highest rates of increase are consistently seen in the 16-24 year age group, which reflect the substantial number of bacterial STIs seen in the $16-19$ year age group (fig 3). ${ }^{32-36}$

Hospital inpatient data consist of acute cases, women experiencing recurrent chronic pain, and long term reproductive health problems associated with PID. ${ }^{34}$ Consequently,

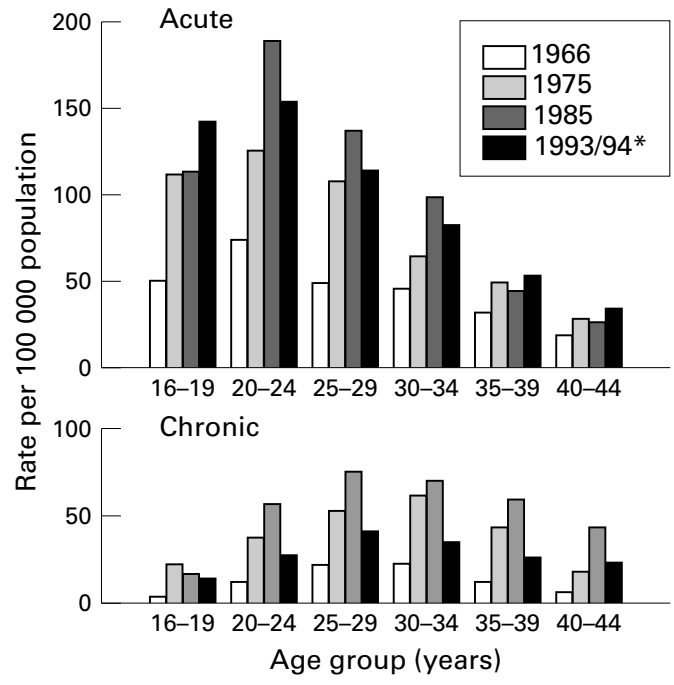

Figure 3 Pelvic inflammatory disease by age group, hospital inpatients England and Wales: 1966-1993/94. these data will not be representative of the true reservoir of PID in the general population. ${ }^{33} 34$ Evidence to support this view comes from reports of ectopic pregnancy and the surveillance of PID in general practice. The incidence of ectopic pregnancy does not reflect the number of PID cases seen in the hospital inpatient admissions data. The stable ectopic pregnancy incidence of $1 / 100$ conceptions (approximately 8000 cases per annum) seen in England is similar to that reported in other European countries. ${ }^{37}$ To sustain this incidence, it would be expected that at least 72000 PID cases would occur annually, assuming 9\% of women with a history of PID develop ectopic pregnancy. ${ }^{10}$ Of course, this underestimates PID prevalence substantially as not all women with a history of PID become pregnant. In fact general practice data suggest that 165000 cases occur every year in reproductive age women, a prevalence of $1.7 \% .^{38}$ Although the PID case definition is likely to have varied between clinical settings, these observations suggest the presence of a substantial reservoir of undiagnosed PID in primary care. The $41 \%$ increase in PID diagnoses seen in attendances in general practice between 1982 and 1992 suggests that PID may be increasingly managed in this setting, although this rise may also reflect increased case ascertainment. ${ }^{39}$ Primary care thus provides an important focus for the diagnosis and treatment of PID; information derived from this setting provides a more complete view of PID epidemiology than hospital inpatient admissions, but diagnosis in primary care is likely to be less specific than in hospital.

\section{Factors associated with PID}

Risk factor studies can identify population subgroups at increased risk of PID, can be used to initiate timely, effective, intervention, and help formulate health education strategies. Risk factors for PID development are closely associated with those of STI acquisition. ${ }^{40}$ Aspects of sexual behaviour, such as age at first sexual intercourse, number of lifetime sexual partners, frequency of partner change, and unsafe sex are key determinants of STI transmission. Age at first sexual intercourse and the number of lifetime sexual partners are known to vary with marital status, cohabitation, and socioeconomic group. ${ }^{41}$ The relation between PID and socioeconomic status is likely to be a surrogate marker of sexual behaviour. Young people are behaviourally vulnerable to STI acquisition as they generally have higher numbers of sexual partners and a higher frequency of partner change than older age groups. ${ }^{41}$ In addition, high PID rates in women aged 16-24 years could reflect longer duration of chlamydial infection or reduced clearance of chlamydial infection in younger women. This could be due to increased host susceptibility, such as a lower concentration of protective chlamydial antibodies, larger cervical ectopy, and greater permeability of cervical mucus than in older age groups. ${ }^{40} 42$

A number of factors have been associated with PID. IUD insertion and termination of pregnancy have been associated with iatrogenic 
PID, which occurs when instrumentation facilitates the introduction of vaginal and cervical micro-organisms into the endometrial cavity. ${ }^{43}$ Cigarette smoking has been associated with increased risk of PID. ${ }^{45}{ }^{46}$ Smoking is thought to either compromise the immune response to infection or the activity of oestrogen. ${ }^{47} 48$ It is also likely that smoking reflects poor health seeking behaviour in lower socioeconomic groups. The association between PID and oral contraceptive (OC) use is also complex and incompletely understood. Although OC use has been associated with a $50 \%$ decrease in PID in reported studies, it is unclear whether OC use prevents ascending infection or protects against symptomatic infection. ${ }^{49}$ Alternatively, both cigarette smoking and OC use may simply be confounding factors that reflect higher sexual risk. Douching has been associated with PID as it is thought to alter the microbiological environment of the vagina and flush bacteria into the uterus..$^{50-52}$ However, although douching is common among women in the United States, less than $0.25 \%$ of UK women report this behaviour and thus it is unlikely that douching is an important factor associated with PID in the United Kingdom. ${ }^{4151}$

It would be unwise to extrapolate the findings of risk factor studies from other countries to England and Wales as sexual health behaviour and contraceptive practice vary between countries and over time. In England and Wales, higher risk of PID has been associated with age 15-34, marital status, lower socioeconomic group, and a history of smoking. ${ }^{37}$ These observations were, however, based on a prevalence study which did not use a consistent case definition, did not take sexual behaviour into account and did not evaluate disease aetiology. ${ }^{38}$ Consequently, although the study represents a starting point for epidemiological investigations, it should be treated with caution and cannot be used as the basis for planning and intervention strategies. Factors associated with PID in England and Wales are thus unknown and properly conducted studies are needed urgently.

Disease burden (industrialised countries) PID accounts for $94 \%$ of morbidity in women associated with STI (including HIV) in established market economies (EME). ${ }^{53}$ The burden of PID among women, measured in terms of disability adjusted life years, was also higher than the burden of disease associated with HIV among men. ${ }^{53}$ This may appear strange as HIV infection causes a substantial burden of mortality and morbidity among homosexual and bisexual men in EMEs. However, although PID is not associated with high mortality ${ }^{54}$ it is associated with high morbidity. The absence of validation studies and an explanation of how these data were derived makes interpretation difficult. Nevertheless, the data indicate that PID is responsible for a considerable disease burden and represents an important healthcare issue in industrialised countries.

\section{Costs associated with PID}

In terms of economic cost, both PID and its sequelae are expensive to individuals, health care systems, and economies. These costs have increased substantially since the development of assisted reproduction techniques such as in vitro fertilisation. In 1992, the cost of a subfertility service in one health district in England and Wales with a population of 46000 women aged 20-44 years were estimated to be $£ 0.88$ million, a national total of $£ 75$ million. ${ }^{55}$ Ten per cent ( $£ 7.5$ million) of this cost was likely to be associated with genital chlamydial infection and thus could have been prevented (fig 1). The economic impact of PID has yet to be evaluated in the United Kingdom. In the United States, direct and indirect costs associated with PID and its sequelae were estimated at over $\$ 4.2$ billion in 1990 and projected to exceed $\$ 10$ billion by the year 2000 , assuming a constant incidence ${ }^{56}$ However, the economic burden associated with PID may have been underestimated as the true incidence of PID is unknown.

\section{Potential for health gain}

The high burden of PID in industrialised countries together with the associated high healthcare costs indicate that there are substantial health gains to be made from the prevention of PID and its sequelae. There are three approaches to effective disease control: education and behavioural change, screening for asymptomatic disease, and diagnosis and treatment of symptomatic disease. Since a substantial proportion of PID cases are chlamydial in origin, a large proportion of cases are potentially preventable through chlamydial intervention. The high level of asymptomatic genital chlamydial infection emphasises the role of screening for this infection.

Primary prevention, based on education and behavioural change, is fundamental to disease control. Behavioural change such as the increased use of barrier contraception and delayed sexual debut in response to HIV and STI health campaigns has been documented in European countries and some have been associated with reduced incidence of symptomatic PID. ${ }^{30} 49558$ However, in the United Kingdom there is a low awareness of PID among healthcare professionals and the public which represents an obstacle to primary prevention. Secondary prevention, or the diagnosis and treatment of asymptomatic genital chlamydial infection, has been successful in reducing both the prevalence of genital chlamydial infection and associated PID. The only randomised controlled trial that has looked at the effectiveness of chlamydial screening indicated that decreases in the prevalence of genital chlamydial infection brought about reductions in PID prevalence. ${ }^{59}$ In the United States, intervention based on screening for genital chlamydial infection has also reduced the incidence of PID and ectopic pregnancy by more than $50 \%$ and $20 \%$ respectively. ${ }^{60}$ Swedish data also indicate that screening for genital chlamydial infection rapidly reduces the incidence of ectopic 
pregnancy among 20-24 year olds. ${ }^{61}$ No study has demonstrated that genital chlamydial screening can reduce the prevalence of tubal factor infertility.

The prevention of the substantial costs associated with PID and related sequelae is one of the key benefits to be gained from screening for genital chlamydial infection. A number of theoretical studies have attempted to quantify the cost effectiveness of such a screening programme. Based on various assumptions, the threshold prevalence of genital chlamydial infection at which chlamydial screening becomes cost effective has been estimated to be between $3.9 \%$ and $6 \%$ (using DNA amplification tests and azithromycin treatment). Threshold prevalences as high as $14 \%$ have also been suggested. ${ }^{62}{ }^{63}$ One reason for this wide variation is that many studies only take the burden of symptomatic PID into account. Sensitivity analysis indicates that a key determinant in the assessment of chlamydial screening cost effectiveness is the prevalence of PID. ${ }^{62}$ If studies are extended to include subclinical or undiagnosed PID, the threshold prevalence at which screening is cost effective may be at least as low as $3.9 \%$. This emphasises the importance of accurately estimating PID prevalence and incidence. Tertiary prevention, the prompt recognition and treatment of symptomatic PID, is also required to prevent repeat episodes and further sequelae. Although antibiotic prophylaxis before either IUD insertion or termination of pregnancy is considered to both reduce the risk of iatrogenic PID and to be cost effective, this evidence is not based on double blinded, randomised controlled trials. ${ }^{44} 64$

Broad spectrum antibiotic treatment will only treat symptomatic PID effectively and prevent sequelae if PID is recognised early. "Silent" or unrecognised PID, a term given to cases of tubal factor infertility with no history of PID, is thought to be characteristic of chlamydial PID and adds to the problems of effective diagnosis. ${ }^{22}$ However, it has also been suggested that unrecognised PID is a result of low diagnostic sensitivity. ${ }^{22}$ Prevention of reexposure to infection through partner notification is another integral part of PID management. However, although this is routinely undertaken in genitourinary medicine clinics, less than a quarter of general practitioners undertake partner notification in suspected PID cases. ${ }^{65}{ }^{66}$ Healthcare professionals need to recognise disease symptoms, promote timely self referral to treatment centres, and encourage therapy compliance among both women and their partners. In the United Kingdom, PID management guidelines have been published by a variety of professional bodies but their impact is difficult to assess. ${ }^{67} 68$ Primary care must play a key part in intervention as the burden of PID appears to be in general practice. Available studies in primary care indicate that PID diagnosis and management varies substantially and is below acceptable standards. ${ }^{66}$ This represents a missed opportunity in the control and prevention of this important source of reproductive ill health.
Pelvic inflammatory disease-key

epidemiological research priorities

- Develop case definition for use in epidemiological research

- Establish social, behavioural, and demographic factors associated with PID

- Estimate disease prevalence/incidence

- Improve surveillance in a range of primary care settings

- Establish diagnostic and management guidelines for use in patient management systems

- Implement validated, representative, active sentinel surveillance

- Estimate the proportion of cases that could be prevented by chlamydial intervention

Professional and public education is required to improve knowledge, attitudes, and skills to ensure effective case management.

\section{Future research priorities}

Epidemiological and surveillance data are crucial to effective disease control as they provide an evidence base for public health action: to define those at risk, to set priorities, plan interventions, and allocate resources. However, available data for England and Wales are clearly biased and insufficient to answer these questions, as they are for many other countries. Since the burden of disease associated with PID is likely to be higher than currently thought, this represents a fundamental gap in our knowledge of STI epidemiology. It prevents a true realisation of the burden of reproductive morbidity among women and the development of an evidenced based approach to the provision of GUM services. In addition, the recent report by the CMO's expert advisory group on $C$ trachomatis highlighted the urgent need for information concerning PID epidemiology, particularly in the assessment of intervention programmes aimed at genital chlamydial infection. ${ }^{2}$ Monitoring the prevalence of genital chlamydial infection could be used to assess such a programme. However, it is potentially biased since, although the prevalence of chlamydial infection may be reduced in the short term by screening, this would not necessarily reflect a corresponding decrease in PID prevalence. Short term reductions in prevalence may be associated with reduced duration of infection rather than reduced disease incidence. Thus, it is only by using PID as the end point measure that the true reproductive health impact of intervention can be measured. ${ }^{3}$

New methods of monitoring PID are urgently required (see box), but a number of methodological issues have to be addressed before epidemiological studies can be undertaken. These problems are not new; many, such as the lack of a simple, specific diagnostic method, variations in reporting practice, and reliance on small scale studies, were described by Weström in $1980 .{ }^{10}$ The fundamental prob- 
lem in PID research is concerned with case definition and diagnostic accuracy. Laparoscopy is considered the definitive diagnostic tool in patient management and academic papers but it is an invasive, expensive, and potentially harmful procedure. In addition, the reproducibility of laparoscopy between clinicians has never been tested, thus the comparability of results between clinicians is open to question. The "gold standard" status of laparoscopy has been questioned ${ }^{69}$ and other invasive techniques, such as plasma cell endometritis, have been suggested to improve PID diagnosis. ${ }^{70}$ However, in the quest to resolve the diagnostic problems posed by PID, tests are becoming increasingly invasive. Such techniques may improve individual patient care but will not improve our knowledge of PID epidemiology as the use of invasive techniques has resulted in predominantly small scale, unrepresentative studies that have inherent selection and participation biases. Non-invasive techniques such as ultrasound have been used as PID diagnostic tools but there is insufficient evidence to assess the value of ultrasound, particularly in the context of atypical presentation. Magnetic resonance imaging has also been used as a PID diagnostic tool and is considered to be more accurate than transvaginal ultrasonography. ${ }^{71}$ However, both techniques use equipment not generally available in primary care settings and thus could not be used for large scale diagnostic testing.

PID epidemiological studies have to move away from a reliance on invasive diagnostic techniques towards syndromic diagnosis which can be used in a variety of clinical settings, including primary care. While this may not offer a perfect solution in terms of diagnosis, as it would compromise diagnostic accuracy, it would allow representative studies to be undertaken in a variety of clinical settings. A syndromic diagnosis would be based on a simple algorithm consisting of easily ascertained clinical variables and the elimination of competing diagnoses. While such definitions have been available for a number of years, their use in epidemiological research has been limited. ${ }^{72-74}$ Syndromic diagnosis could be incorporated within established PID management guidelines and tailored for use within primary care. Existing computer based patient management systems could be used to help ensure standard delivery of care. However, the use of syndromic diagnosis is controversial: its use in the control of STIs in developing countries has been disappointing and any PID diagnostic algorithm would be difficult to validate in terms of sensitivity and specificity. However, overdiagnosis and overtreatment are seen as being acceptable in the management of suspected PID as they ensure appropriate antibiotic therapy earlier in the course of disease and consequently will be more effective in preventing future reproductive morbidity. ${ }^{75}$

The evaluation of risk factors associated with PID presents particular problems. Many studies have been based on small sample sizes and undertaken over several years. This makes results hard to interpret as aetiology varies over time and associated risk factors vary with the aetiology. ${ }^{76}$ Ideally, risk factor studies should be representative, be undertaken over a short time, and should evaluate sexual behaviour. A case-control study is the most efficient and cost effective method of undertaking such studies. This is particularly relevant in view of the time constraints and high costs associated with large scale microbiological and immunological testing. However, there are a number of problems specifically associated with a case-control study investigating risk factors for PID, and the selection of both cases and controls is difficult. Again, use of a laparoscopic gold standard diagnosis for cases presents a problem. Few women with a clinical diagnosis of PID undergo a laparoscopy, and it is considered unethical to undertake laparoscopy if a competing diagnosis is not suspected and/or there is no need to alleviate symptoms. The dilemma researchers are then faced with is should a syndromic diagnosis be used or should a more biased group of laparoscoped cases be used. Control group selection is also difficult. Ideally, the control group should be taken from a randomly selected group of women of child bearing age representative of the population from which cases were derived. In addition, to ensure there were no cases of PID among the controls, all controls would need to undergo laparoscopy. Women requesting laparoscopic sterilisation would fit these criteria but are likely to be a biased control group. Parity is likely to be a factor associated with PID but those attending for laparoscopic sterilisation are likely to have higher parity, on average, than the general population. This would lead to a biased view of the odds ratios associated with various factors such as the number of pregnancies, contraceptive use, and sexual behavioural measures. There is no perfect solution to these problems but risk factor data are needed and consequently compromises in study design would be necessary.

\section{Conclusions}

PID is a key issue facing women's reproductive health in England and Wales and many other countries. It is clear that the available data do not provide an accurate view of PID epidemiology and assumptions cannot be made based on these data. This review has evaluated the priority areas for epidemiological research which will create an evidence base for intervention and control. Such research is urgently required as PID remains the most important, preventable STD in industrialised countries: its impact is only just being recognised and control remains elusive.

We would like to thank Caroline Akehurst and the editorial board of Eurosurveillance for their help in assessing the availability of European PID surveillance datasets, Dr M Sutton (CDC Atlanta) for US data, and Professor J Paavonen and Dr P E Munday for their helpful suggestions on the content of this review.

Contributors: IS instigated the project and wrote the first draft; JS made a substantial contribution to the structure and content of the review.

1 Pearce J. Pelvic inflammatory disease. BMF 1990;300:1090- 
2 Department of Health. Summary and conclusions of CMO' Expert Advisory Group. London: DoH, 1998.

3 Simms I, Hughes G, Catchpole M. Screening for Chlamydia trachomatis. BMF 1998;317:680-1

4 Sweet R. Microbial etiology of pelvic inflammatory disease. In: Landers D, Sweet R, eds. Pelvic inflammatory disease. New York: Springer-Verlag, 1996.

5 Eschenbach D, Hillier S, Critchlow C, et al. Diagnosis and clinical manifestations of bacterial vaginosis. Am f Obstet Gynecol 1988;158:819-28.

6 Sweet RL. Role of bacterial vaginosis in pelvic inflammatory disease. Clin Infect Dis 1995;20:S271-5.

7 Brunham RC, Binns B, Guijon F, et al. Etiology and outcome of acute pelvic inflammatory disease. F Infect $D i$ 1988;158:510-17.

8 Wølner-Hanssen P, Paavonen J, Kiviat N, et al. Outpatien treatment of pelvic inflammatory disease with cefoxitin and doxycycline. Obstet Gynecol 1988;71:595-600.

9 Bevan C, Johal B, Mumtaz G, et al. Clinical, laparoscopic and microbiological findings in acute salpingitis: report on a United Kingdom cohort. Br F Obstet Gynaecol 1995;102: 407-14.

10 Weström L. Incidence, prevalence and trends of acute pelvic inflammatory disease and its consequences in industrialised countries. Am 7 Obstet Gynecol 1980;138:880-92.

11 Buchan H, Vessey M, Goldacre M, et al. Morbidity following pelvic inflammatory disease. Br f Obstet Gynaecol 1993; 100:558-62.

12 Risch H, Howe G. Pelvic inflammatory disease and the risk of epithelial ovarian cancer. Cancer Epidemiol Biomarkers Prev 1995;4:447-51.

13 Cohen C, Brunham R. Pathogenesis of chlamydia induced pelvic inflammatory disease. Sex Transm Inf 1999;75:21-4.

14 Toye B, Laferrière $\mathrm{P}$, Claman $\mathrm{P}$, et al. Association between antibody to the chlamydial heat-shock protein and tubal infertility. F Infect Dis 1993;168:1236-40.

15 Brunham R, Peeling R, Maclean I, et al. Chlamydia trachomatis-associated ectopic pregnancy: serologic and histologic correlates. F Infect Dis 1992;165:1076-81.

16 Paavonen J, Lehtinen M. Immunopathogenesis of chlamydial pelvic inflammatory disease: the role of heat-shock proteins. Infect Dis Obstet Gynecol 1994;2:105-10.

17 Korn A, Landers D. Pelvic inflammatory disease and HIV-1 infection. In: Landers D, Sweet R, eds. Pelvic inflammatory disease. Pittsburgh: Springer, 1999 .

18 Stamm W, Guinan M, Johnson C, et al. Effect of treatment regimens for Neisseria gonorrhoeae on simultaneous infection with Chlamydia trachomatis. N Engl f Med 1984;310 $545-9$

19 Weström L. Sexually transmitted diseases and infertility. Sex Transm Dis 1994;21:S32-7.

20 Swenson C, Schachter J. Infertility as a consequence of chlamydial infection of the upper genital tract in female mice. Sex Transm Dis 1984;11:64-7.

21 Hillis S, Joesoef R, Marchbanks P, et al. Delayed care of pelvic inflammatory disease as a risk factor for impaired fertility. Am 7 Obstet Gynecol 1993;168:1503-9.

22 Wølner-Hanssen P. Silent pelvic inflammatory disease: is it overstated? Obstet Gynecol 1995; 86:321-5.

23 Howard M, Doherty J, Zimic-Vincentic M, et al. An epidemiologic study of a decrease in hospitalizations for pelvic inflammatory disease (PID) in two Canadian cities (1985 1995). Ninth International Symposium on Human Chlamydial Infection, Napa Valley, USA, 1998.

24 Kamwendo F, Forslin L, Bodin L, et al. Decreasing incidences of gonorrhoea and chlamydia associated acute pelvic inflammatory disease. Sex Transm Dis 1996;23:384 91.

25 Kamwendo F, Forslin L, Bodin L, et al. Programmes to reduce pelvic inflammatory disease - the Swedish experience. Lancet 1998;351:25-8.

26 Simms I, Catchpole M, Brugha R, et al. Epidemiology of genital Chlamydia trachomatis in England and Wales. Genitourin Med 1996;73:122-6.

27 Hiltunen-Back E, Rostila T, Kautiainen H, et al. Rapid decrease of endemic gonorrhea in Finland. Sex Transm Di 1998;25:181-6.

28 Hansson H, Danielsson D. Epidemiology of sexually transmitted diseases in the Scandinavian countries. Scand $f$ Infect Dis 1982;32:149-56.

29 Meirik O. Ectopic pregnancy during 1961-78 in Uppsala County, Sweden. Acta Obstet Gynaecol Scand 1981;60:545-

30 Weström L. Decrease in incidence of women treated in hospital for acute salpingitis in Sweden. Genitourin Med 1988; 64:59-63.

31 Eschenbach D, Harnisch J, Holmes K. Pathogenesis of acute pelvic inflammatory disease: role of contraception and other risk factors. Am f Obstet Gynecol 1977;128:838 50 .

32 Hughes G, Simms I, Rogers P, et al. New cases seen a genitourinary medicine clinics: England 1997. CDR Suppl 1998;8:S1-12.

33 Robinson N, Beral V, Ashley J. Trends in pelvic inflammatory disease in England and Wales. F Epidemiol Commun Health 1981;35:265-70.

34 Buchan H, Vessey M. Epidemiology and trends in hospital discharges for pelvic inflammatory disease in England, 1975 to 1985. Br f Obstet Gynaecol 1989;96:1219-23.

35 Adler $M$. Trends for gonorrhea and pelvic inflammatory disease in England and Wales and for gonorrhea in defined population. Am f Obstet Gynecol 1980;138:901-4.

36 Department of Health. Hospital episode statistics. London: HMSO, 1994.
37 Simms I, Rogers P, Nicoll A. The influence of demographic change and cumulative risk of pelvic inflammatory disease on the incidence of ectopic pregnancy. Epidem Infect 1997; 119:49-52

38 Simms I, Rogers P, Charlett A. The rate of diagnosis and demography of pelvic inflammatory disease in general practice: England and Wales. Int F STD AIDS 1999;10: 448-51.

39 OPCS. Morbidity statistics from general practice: fourth national study, 1991-1992. London: HMSO, 1995.

40 Washington A, Aral S, Wølner-Hanssen P, et al. Assessing risk for pelvic inflammatory disease and its sequelae. fAMA 1991;266:2581-6.

41 Johnson A, Wadsworth J, Wellings K, et al. Sexual attitudes and lifestyles. Oxford: Blackwell Scientific, 1994.

42 Cates Jr W, Rolfs Jr R, Aral S. Sexually transmitted diseases, pelvic inflammatory disease, and infertility: an epidemiologic update. Epidemiol Rev 1990;12:199-220.

43 Vessey M, Doll R, Peto P. A long term follow-up study of women using different methods of contraception-an interim report. f Biosoc Sci 1976;8:373-426.

44 Blackwell A, Thomas P, Wareham K, et al. Health gains from screening for infection of the lower genital tract in women
attending for termination of pregnancy. Lancet 1993;342: attending

45 Marchbanks P, Lee N, Petersen H. Cigarette smoking as a risk factor for pelvic inflammatory disease. $\mathrm{Am} \mathcal{F}$ Obste Gynecol 1990;162:639-44

46 Scholes D, Daling J, Stergachis A. Current cigarette smoking and risk of acute pelvic inflammatory disease. $A m$ f Public Health 1992;82:1352-5.

47 Hersey P, Prendergast D, Edwards A. Effects of cigarette smoking on the immune system: follow-up studies in normal subjects after cessation of smoking. Med $\mathcal{f}$ Aust 1983;2:425-9.

48 Pasley J, Rank R, Hough AJ. Effects of various doses of estradiol on chlamydia genital infection in ovariectomized guinea pigs. Sex Transm Dis 1985;12:8-13.

49 Wølner-Hanssen P, Eschenbach D, Paavonen J. Decreased risk of symptomatic chlamydial pelvic inflammatory disease associated with oral contraception use. $7 A M A$ 1990;263:54-9.

50 Scholes D, Daling J, Stergachis A, et al. Vaginal douching as a risk factor for acute pelvic inflammatory disease. Obstet Gynecol 1993;81:601-5.

51 Forrest K, Washington A, Daling J, et al. Vaginal douching as a possible risk factor for pelvic inflammatory disease. 7 Nat Med Asoc 1989;81:159-65.

52 Wølner-Hanssen P, Eschenbach D, Paavonen J, et al. Association between vaginal douching and acute pelvic inflammatory disease. $\mathcal{F} A M A$ 1990;263:1936-41.

53 World Bank. World development report 1993. Investing in health. World development indicators. New York: Oxford University Press, 1993.

54 OPCS. Mortality statistics, cause: England and Wales, 1992. London: HMSO, 1992

55 School of Public Health UoL. Effective health care: the management of subfertility. Leeds: University of Leeds, 1992.

56 Washington A, Katz P. Cost of and payment source for pelvic inflammatory disease. $\mathcal{F} A M A$ 1991;226:2565-9.

57 Evans B, Catchpole M, Heptonstall M, et al. Sexually transmitted diseases and HIV-1 infection among homosexua men in England and Wales. BMF 1993;306:426-8.

58 Coutinho R, Rijsdijk A, van den Hoek J. Decreasing incidence of PID in Amsterdam. Genitourin Med 1992;68: 353-5.

59 Scholes D, Stergachis A, Heidrich F, et al. Prevention of pelvic inflammatory disease by screening for cervical chlamydial infection. N Engl ₹ Med 1996;334:1362-6.

60 Hillis S, Nakashima A, Amsterdam L, et al. The impact of a comprehensive chlamydia prevention program in Wisconcomprehensive chlamydia prevention p

61 Egger M, Low N, Smith G, et al. Screening for chlamydia infections and the risk of ectopic pregnancy in a county in Sweden: ecological analysis. BMF 1998;316:1776-80.

62 Paavonen J, Puolakkainen M, Paukku M, et al. Cost-benefit analysis of first-void urine Chlamydia trachomatis screening program. Obstet Gynecol 1998;92:292-8.

63 Genc M, Mårdh P. A cost-effectiveness analysis of screening and treatment for Chlamydia trachomatis infection in asymptomatic women. Ann Intern Med 1996;124:1-7.

64 Sinei S, Schultz K, Lamptey P, et al. Preventing IUCDrelated pelvic infection: the efficacy of prophylactic doxycy-

65 Robinson A, Greenhouse P. Prevention of recurrent pelvic infection by contact tracing: a commonsense approach. $B$ f Obstet Gynaecol 1996;103:859-61.

66 Simms I, Vickers M, Stephenson J, et al. National assessment of PID diagnosis, treatment and manag in general practice: England and Wales. Thirteenth Meeting of the International Society for Sexually Transmitted Diseases Research. Denver, USA, 1999. Abstract No 305

67 Templeton A. The prevention of pelvic infection. London: RCOG Press, 1996.

68 CDC. 1998 Guidelines for treatment of sexually transmitted diseases. $M M W R$ 1998;47:1-119.

69 Sellors J, Mahony J, Goldsmith C, et al. The accuracy of clinical findings and laparoscopy in pelvic inflammatory disease. Am 7 Obstet Gynecol 1991;164:113-20.

70 Korn A, Hessol N, Padian N, et al. Commonly used diagnostic criteria for pelvic inflammatory disease have 
poor sensitivity for plasma cell endometritis. Sex Transm Dis 1995:22:335-41.

71 Tukeva T, Aronen H, Karjalalnen P, et al. MR imaging in pelvic inflammatory disease: comparison with laparoscopy amd US. Radiology 1999;210:209-16.

72 Hager W, Eschenbach D, Spence M, et al. Criteria for diagnosis and grading of salpingitis. Obstet Gynecol 1983;61: 113-14.

73 Jacobson L, Weström L. Objectivised diagnosis of acute pelvic inflammatory disease. Am f Obstet Gynecol 1969;105: 1088-98.

74 Hadgu A, Weström L, Brookes C, et al. Predicting acute pelvic inflammatory disease: a multivariate analysis. $A m \mathscr{F}$ vic inflammatory disease: a must

75 Rolfs R. "Think PID”. Sex Transm Dis 1991:18:131-2.

76 Jossens M, Schachter J, Sweet R. Risk factors associated with pelvic inflammatory disease of differing microbial etiologies. Obstet Gynecol 1994;83:989-97.

77 Kinghorn G, Duerden B, Hafiz S. Clinical and microbiological investigation of women with acute salpingitis and their consorts. Br f Obstet Gynaecol 1986;93:869-80.

78 Stacey C, Munday P, Taylor-Robinson D, et al. A longitutdinal study of pelvic inflammatory disease. $\mathrm{Br} F \mathrm{Obstet}$ Gynaecol 1992;99:994-9.

79 Paavonen J. Chlamydia trachomatis in acute salpingitis. Am f Obstet Gynecol 1980;138:957-9.

80 Heinonen P, Teisala K, Miettinen A, et al. A comparison of ciprofloxacin with doxycycline plus metronida ciproxact of acute pelvic inflammatory disease. Scand the Infect 1989;60:66-73.
81 Paavonen J, Teisala K, Heinonen P, et al. Microbiological and histopathological findings in acute pelvic inflammatory disease. Br F Obstet Gynaecol 1987;94:454-60.

82 Ripa KT, Svensson L, Treharne J, et al. Chlamydia trachomatis infection in patients with laparoscopically verified acute salpingitis. Am f Obstet Gynecol 1980;138:960-4.

83 Brihmer C, Kallings I, Nord C-E, et al. Salpingitis: aspects of diagnosis and etiology: a 4-year study from a Swedish capital hospital. Eur f Obstet Gynecol Reprod Biol 1987;24: capital hosp $211-20$.

84 Soper D, Brockwell N, Dalton H, et al. Observations concerning the microbial etiology of acute salpingitis. $A m \mathcal{F}$ Obstet Gynecol 1994;170:1008-14.

85 Cacciatore B, Leminen A, Ingman-Friberg $\mathrm{S}$, et al. Transvaginal sonographic findings in ambulatory patients with suspected pelvic inflammatory disease. Obstet Gynecol 1992;80:912-16

86 Wasserheit J, Bell T, Kiviat N, et al. Microbial causes of proven pelvic inflammatory disease and efficacy of proven pelvic inflammatory disease and efficacy of 187-93.

87 Kiviat N, Wølner-Hanssen P, Peterson M, et al. Localization of Chlamydia trachomatis infection by direct immunofluorescence and culture in pelvic inflammatory disease. $\mathrm{Am}$ Obstet Gynecol 1986;154:865-73.

88 Livengood C, Hill G, Addison W. Pelvic inflammatory disease: findings during inpatient treatment of clinically severe, laparoscopy-documented disease. Am 7 Obstet Gynecol 1992;166:519-24. 